\title{
THE CARDIOVASCULAR AND RESPIRATORY EFFECTS OF ISOFLURANE-NITROUS OXIDE ANAESTHESIA
}

\author{
William M. Dolan, m.D. $\nmid$, Wendell C. Stevens, m.D., \\ Edmond I. Eger, II, M.D., "Thomas H. Cromwell, M.D., \\ Michael J. Halsey, Ph.D., "Thomas F. Shakespeare, M.D.," \\ aNd Ronald D. MiLler, M.D. ${ }^{\circ}$
}

\section{INTRODUCTION}

IsoflunaNe (Forane ${ }^{\circledR} 1$ chloro-2-2-2 trifluoroethyl, difluoromethyl ether), is a halogenated ether currently being evaluated for use as an inhalational anaesthetic. Its favourable properties include non-flammability, ${ }^{1}$ relatively low blood and fat solubilities (blood-gas and oil-gas partitions of 1.4 and 99 , respectively), ${ }^{1,2}$ molecular stability resulting in resistance to dehydrohalogenation and hepatic metabolism, ${ }^{1,3}$ lack of sensitization of the heart to catecholamines, ${ }^{4}$ and favourable depression of neuromuscular function and potentiation of muscle relaxants. ${ }^{5}$ However, isoflurane can produce profound depression of ventilation and blood pressure $^{B, 7}$ Since the concurrent use of nitrous oxide with halothane attenuates the hypotension and ventilatory depression caused by halothane, ${ }^{8,9,10,11}$ we asked whether nitrous oxide would produce similar changes towards awake values during isoflurane anaesthesia.

\section{METHODS}

We studied the cardiovascular and respiratory effects of anaesthesia with isoflurane and 70 per cent nitrous oxide in eight healthy, unpremedicated volunteers. The volunteers were $25 \pm 1$ (SD) years of age and were informed of the purposes, procedures, and hazards of the study. The protocol was approved by the Committee on Human Experimentation of the University of California, San Francisco. A normal medical history, physical examination, chest roentgenogram, complete blood count, serum glutanic oxaloacetic transaminase and lactic dehydrogenase were required before a volunteer was accepted for anaesthesia.

Our methods have been described previously. ${ }^{12}$ Briefly, radial artery, right atrial and forearm venous catheters were placed under local anaesthesia for measurement of arterial, right atrial and peripheral venous pressures, arterial and central venous blood gases $\left(\mathrm{PO}_{2}, \mathrm{PCO}_{2}\right.$, and $\left.\mathrm{pH}\right)$, haematocrit, cardiac output, and blood volume (the latter two by dye-dilution techniques). Oxygen consump-

From the Departments of Anaesthesia, University of California, ${ }^{\circ}$ San Francisco, California 94143 and Stanford University Medical Center, Palo Alto, California 94305.†

This work was supported by Program Project No. USPHSI PO1 GM155571-02, NIH Training Grant No. 5T1-PM00063-12, and Ohio Medical Products, Division of Airco, Inc.

${ }^{\circ}$ Trademark, Ohio Medical Products, a Division of Airco, Inc. 
tion was determined by calculating the arterial-to-venous oxygen content difference and multiplying by the cardiac output. Forearm and finger blood flows were measured with Whitney gauges. The electrocardiogram, carotid pulse tracing, and precordial phonocardiogram were recorded continuously. Cardiac pre-ejection periods and ejection times were measured from the electrocardiogram in conjunction with the arterial pressure wave and heart sounds. Ballistocardiograms (I-J wave amplitude) were obtained with the volunteers lying on an ultra-low frequency air bearing ballistocardiogram bed. Skin and oral (awake) or œsophageal (anaesthetized) temperatures were monitored and maintained at normal levels by varying room temperatures. When respiratory measurements were made, we recorded respiratory rate and tidal volumes using a recording ventimeter. ${ }^{13}$ End-tidal $\mathrm{PCO}_{2}$ and isoflurane determinations were made with LB-1 infrared analyzers. For isoflurane analysis, the span was set against a standard in nitrogen. With the zero suppressed with 70 per cent nitrous oxide to eliminate the cross-over effect of nitrous oxide, we calibrated the analyzer with isoflurane in 70 per cent nitrous oxide. Isoflurane concentrations for these calibrations were determined simultaneously by gas chromatography.

Control values for each of these parameters were obtained with the subject awake, breathing a mixture of air with oxygen to give a 30 per cent inspired oxygen concentration. We obtained control values during both controlled and spontaneous ventilation. After a brief practice period, volunteers allowed controlled ventilation with an Air-Shields Ventilator to maintain arterial carbon dioxide tension $\left(\mathrm{PaCO}_{2}\right)$ at a value slightly below that obtained during normal spontaneous ventilation. Serial measurements of cardiovascular variables were made during each ventilatory state, until stable readings were obtained. The two lowest cardiac output and other associated cardiovascular values were considered as representative of the awake, resting state. We also obtained measurements of cardiac and respiratory function before anaesthesia was started, with the volunteers breathing spontaneously and still inspiring 30 per cent oxygen.

Anaesthesia was induced with a mask and conventional anaesthetic circle system. Intubation of the trachea was performed without use of muscle relaxants. Controlled ventilation kept $\mathrm{PaCO}_{2}$ at awake levels. Except for a brief period, inspired nitrous oxide was maintained at 70 per cent. This was measured indirectly by analysis of the inspired oxygen concentrations and subtraction of that value from 100 per cent. Measurements during anaesthesia were divided into early, middle, and late measurements. Over the first one and a half hours of anaesthesia, we obtained an "early" dose response test using $0.65,1.20$, and 1.95 per cent end-tidal isofurane. Cardiovascular measurements were made after at least ten minutes at each dose. After at least five hours of total anaesthesia time, we repeated cardiovascular measurements at the same three isoflurane doses and called this the "late" dose response test.

Between the early and late dose response tests we performed the "middle" measurements. We allowed the subjects to breathe spontaneously and studied respiratory and cardiovascular functions at $0.6,1.3$, and 1.5 per cent end-tidal isoflurane. Then ventilation was controlled again to lower $\mathrm{PaCO}_{2}$ to the awake value, end-tidal isoflurane was adjusted to 1.3 per cent and nitrous oxide was 
discontinued. After twenty minutes of isoflurane and oxygen we obtained another set of cardiovascular measurements. Seventy per cent nitrous oxide was resumed and we performed the late dose response test with controlled ventilation.

All volunteers received infusions of lactated Ringer's solution, or 5 per cent dextrose in water to which $22 \mathrm{mEq}$ of sodium bicarbonate per liter had been added. These solutions were used to clear the catheters following blood sampling. The volumes infused were more than sufficient to replace 300 to $400 \mathrm{ml}$ of blood removed for samples. Serial haematocrits and blood volumes were obtained.

A mean and standard error were determined for all our data. We compared cardiovascular and respiratory measurements when performed at different isoflurane concentrations by paired analysis. We also compared our data with those of two previously reported studies of volunteers during isoflurane-oxygen anaesthesia: seven subjects whose ventilation was controlled ${ }^{6}$ and nine subjects who were allowed to breathe spontaneously. ${ }^{7}$ To compare these data on an equivalent dose scale we computed a MAC multiple for each anaesthetic dose by dividing the isoflurane concentrations by 1.27 which is $\mathrm{MAC}$ in the age group studied. ${ }^{14}$ Seventy per cent nitrous oxide reduces isoflurane requirement by an amount equivalent to 0.75 per cent isoflurane in this age group. Whenever 70 per cent nitrous oxide was present, we added 0.75 to the end-tidal isoflurane before calculations of the MAC multiple. We used the unpaired " $t$ " test for comparison at equivalent MAC multiples, accepting as significant a " $\mathrm{p}$ " value of less than 0.05 .

\section{RESULTS}

Table I lists the physical characteristics of the volunteers and the awake (control) cardiorespiratory values.

Controlled Ventilation (Table II). Arterial pressure and total peripheral resistance decreased with induction of anaesthesia and decreased further as the dose of isoflurane-nitrous oxide was increased. At $2 \mathrm{MAC}$, the blood pressure was 73 per cent of control. The total peripheral resistance had decreased to 65 per cent of the awake value. No significant change in cardiac output or pre-ejection period occurred. Early in anaesthesia the ballistocardiogram I-J wave decreased. Early in anaesthesia there was a 2 to 3 torr elevation of mean right atrial pressure at the two deeper levels of anaesthesia. Metabolic acidosis did not occur. Muscle blood flow increased with onset of anaesthesia and increased further as the anaesthetic dose was increased. The only differences between early and late results were a further increase in muscle blood flow, decrease in right atrial pressure, and a small increase in base excess.

Spontaneous Ventilation (Table III). Isoflurane-nitrous oxide produced a doserelated increase in $\mathrm{PaCO}_{2}$. Although there was a threefold increase of respiratory rate, minute ventilation did not change significantly from awake values because tidal volume decreased. Cardiac output increased as anaesthetic dose increased. Despite increase of cardiac output, arterial pressure still decreased, indicating that a fall in total peripheral resistance also occurred. No significant changes in I-J amplitude, pre-ejection period, or ejection time occurred nor were there sig- 
TABLE I

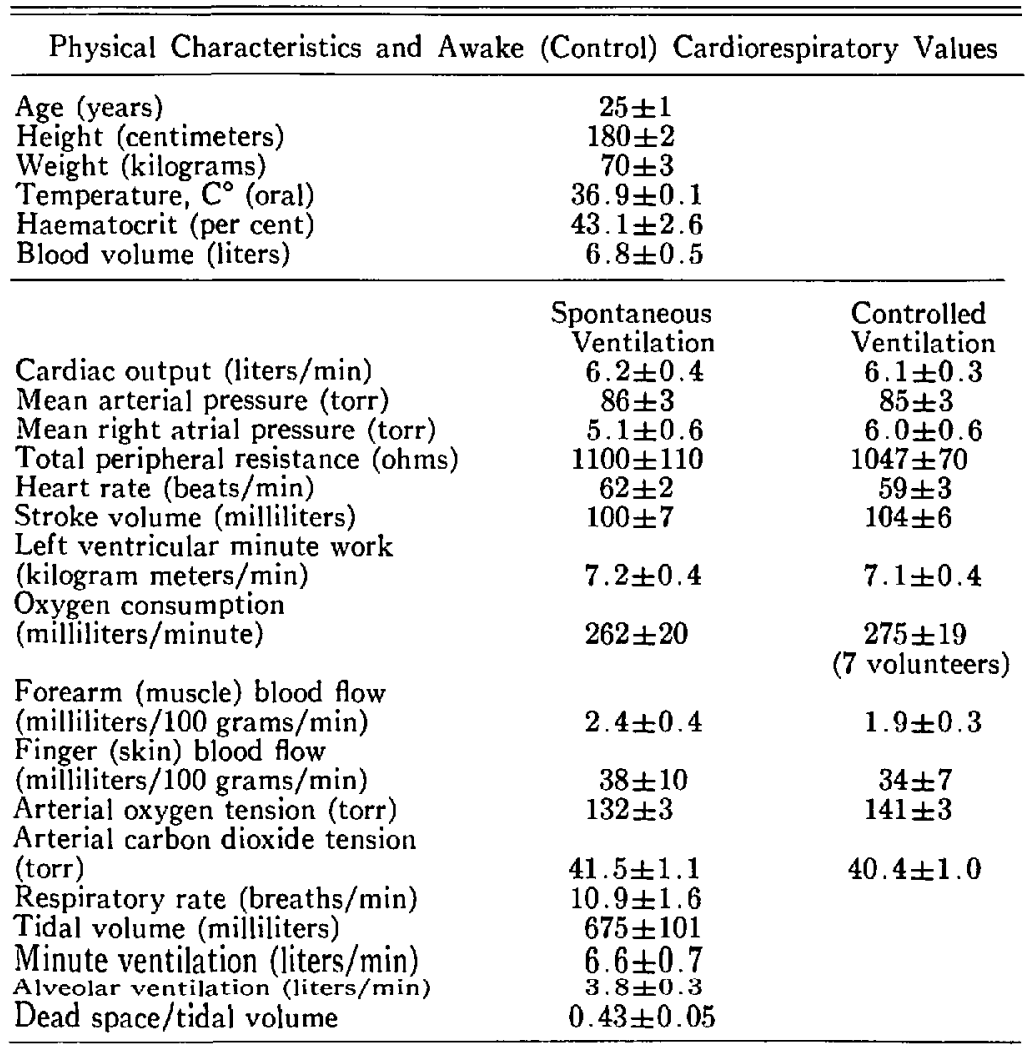

Mean values \pm 1 standard error are given.

nificant elevations of right atrial pressure or base excess. Muscle flows, again, were elevated.

Removal of nitrous oxide decreased heart rate, cardiac output, and I-J amplitude despite a decrease of total anaesthetic dose of over one-half MAC. Total peripheral resistance increased, since arterial pressure was unchanged. Stroke volume also was unchanged.

Average total fluid replacement was $2030 \pm 190 \mathrm{ml}$ (SE). The amount of supplemental bicarbonate each patient received was $8 \pm 3 \mathrm{mEq}$. Blood volume and haematocrit decreased slightly with time (Table IV).

Body temperature was held within narrow limits in all patients. The mean oesophageal temperature was $36.9^{\circ} \mathrm{C}$ with a range of $32.6^{\circ} \mathrm{C}$ to $37.4^{\circ} \mathrm{C}$.

We compared our data with the data from previous investigations during isoflurane-oxygen anaesthesia. Table $\mathrm{V}$ lists the data from four studies: isofluraneoxygen with spontaneous and controlled ventilation ${ }^{6,7,8}$ and the present studies using isoflurane-nitrous oxide with spontaneous and controlled ventilation. Only two levels of anaesthesia were nearly equivalent in terms of MAC multiples in all four studies. Because we had only one dose-response period with isofluranenitrous oxide and spontaneous ventilation and this was at least three hours after induction of anaesthesia, we compared our spontaneous ventilation data with late dose-response periods during isoflurane-oxygen anaesthesia. 
DOLAN, et al.: ISOFLURANE-NITROUS OXIDE ANAESTHESIA

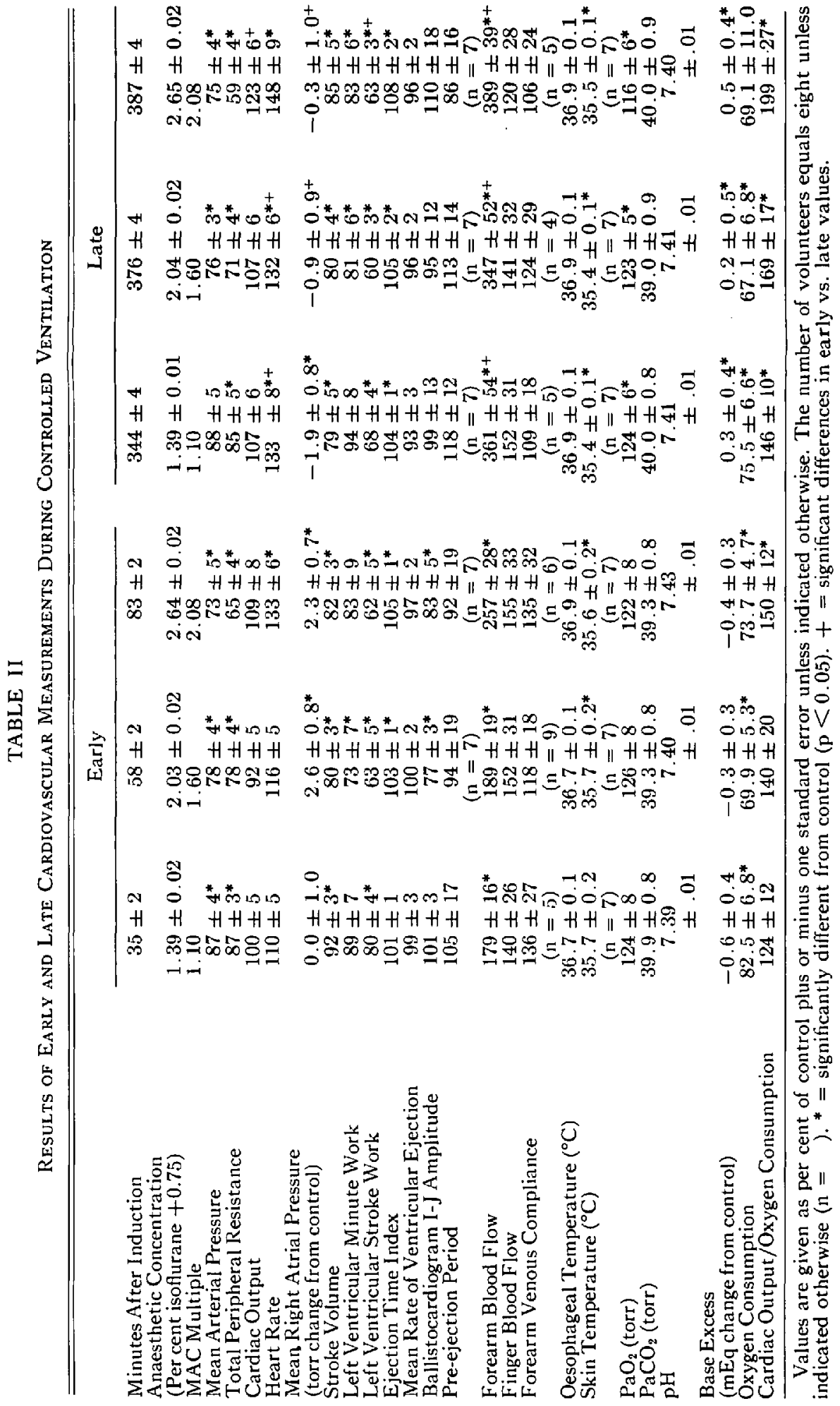


TABLE III

Results of Cardiovascular and Respiratory Measurements During Spontaneous Ventilation (Middle Measurement)

\begin{tabular}{|c|c|c|c|}
\hline Minutes After Induction & $189 \pm 14$ & $213 \pm 5$ & $201 \pm 15$ \\
\hline $\begin{array}{l}\text { Alveolar Anaesthetic Concentration } \\
\text { (per cent isoflurane +0.75) } \\
\text { MAC Multiple }\end{array}$ & $1.38 \pm 0.01$ & $2.02 \pm 0.2$ & $2.27 \pm 0.01$ \\
\hline Minute Ventilation & $\begin{array}{c}113 \pm 7 \\
(n=7)\end{array}$ & $\begin{array}{c}100 \pm 8 \\
(n=7)\end{array}$ & $\begin{aligned} 105 & \pm 7 \\
(n & =6)\end{aligned}$ \\
\hline Respiratory Rate & $240 \pm 40^{*}$ & $\begin{aligned} 280 & \pm 50^{*} \\
(\mathrm{n} & =7)\end{aligned}$ & $\begin{array}{c}330 \pm 70^{*} \\
(n=6)\end{array}$ \\
\hline Tidal Volume & $\begin{array}{l}55 \pm 8^{*} \\
(n=7)\end{array}$ & $\begin{array}{l}40 \pm 6^{*} \\
(\mathrm{n}=7)\end{array}$ & $\begin{array}{l}38 \pm 6^{*} \\
(n=6)\end{array}$ \\
\hline Deadspace/Tidal Volume & $\begin{array}{c}0.65 \pm 0.02 \\
(n=7)\end{array}$ & $\begin{array}{l}0.70 \pm 0.02 \\
(\mathrm{n}=7)\end{array}$ & $\begin{array}{c}0.73 \pm 0.03 \\
(n=7)\end{array}$ \\
\hline Arterial Carbon Dioxide & & & \\
\hline Tension (torr) & $47.3 \pm 1.9^{*}$ & $58.5 \pm 3.2^{*}$ & $67.9 \pm 4.5^{\star}$ \\
\hline Arterial Oxygen Tension (torr) & $111 \pm 7$ & $\begin{array}{r}108 \pm 6 \\
79 \pm 4^{*}\end{array}$ & $\begin{array}{l}92 \pm 8 \\
78+7 *\end{array}$ \\
\hline Cardiac Output & $116 \pm 5^{*}$ & $142 \pm 10^{*}$ & $167 \pm 15^{*}$ \\
\hline Total Peripheral Resistance & $75 \pm 7^{*}$ & $57 \pm 5^{*}$ & $54 \pm 3^{*}$ \\
\hline Heart Rate & $124 \pm 6^{*}$ & $132 \pm 7^{*}$ & $136 \pm 11^{*}$ \\
\hline Heart Rate & $124 \pm 6^{*}$ & $132 \pm 7^{*}$ & $136 \pm 11^{*}$ \\
\hline Stroke Volume & $96 \pm 7$ & $109 \pm 8$ & $124 \pm 7^{*}$ \\
\hline Left Ventricular Minute Work & $94 \pm 5$ & $125 \pm 18$ & $\begin{array}{r}127 \pm 16 \\
(n=7)\end{array}$ \\
\hline Ejection Time Index & $\begin{array}{c}150 \pm 2 \\
(n=7)\end{array}$ & $105 \pm 1$ & $\begin{aligned} 105 & \pm 3 \\
(\mathrm{n} & =6)\end{aligned}$ \\
\hline Pre-ejection Period & $96 \pm 17$ & $99 \pm 25$ & $78 \pm 15$ \\
\hline $\begin{array}{l}\text { Ballistocardiogram I-J Amplitude } \\
\text { Mean Right Atrial Pressure }\end{array}$ & $114 \pm 11$ & $120 \pm 16$ & $122 \pm 17$ \\
\hline (torr change from control) & $-2.2 \pm 1.1$ & $-1.3 \pm 1.0$ & $-1.2 \pm 0.9$ \\
\hline Base Deficit & & & \\
\hline $\begin{array}{l}\text { (mEq change from control) } \\
\text { Oxygen Consumption }\end{array}$ & $\begin{array}{c}+1.6 \pm 0.7 \\
75 \pm 7 \\
(\mathrm{n}=7)\end{array}$ & $\begin{array}{c}+1.9 \pm 0.9 \\
76 \pm 7 \\
(\mathrm{n}=7)\end{array}$ & $\begin{aligned}+1.8 & \pm 1.1 \\
83 & \pm 9\end{aligned}$ \\
\hline Cardiac Output/Oxygen Consumption & $\begin{array}{c}158 \pm 13^{*} \\
(\mathrm{n}=7)\end{array}$ & $\begin{array}{c}201 \pm 26^{*} \\
(\mathrm{n}=7)\end{array}$ & $\begin{aligned} 228 & \pm 49^{*} \\
(\mathrm{n} & =6)\end{aligned}$ \\
\hline Forearm Blood Flow & $292 \pm 37^{*}$ & $334 \pm 62$ & $305 \pm 37^{*}$ \\
\hline Finger Blood Flow & $182 \pm 51$ & $165 \pm 40$ & $204 \pm 82$ \\
\hline Forearm Venous Compliance & $116 \pm 35$ & $97 \pm 32$ & $79 \pm 27$ \\
\hline Oesophageal Temperature $\left({ }^{\circ} \mathrm{C}\right)$ & $36.9 \pm 0.1$ & $36.9 \pm 0.1$ & $36.9 \pm 0.1$ \\
\hline
\end{tabular}

Values are given as per cent of control plus or minus one standard error unless otherwise indicated. The number of volunteers equals eight unless indicated otherwise $(n=){ }^{*}=$ significantly different from control $(p<0.05)$.

\section{DisCUSSION}

\section{Circulatory Effects}

The concomitant use of nitrous oxide and isoflurane produces less hypotension than isoflurane alone. The depression in mean arterial pressure was less for the isoflurane-nitrous oxide group at all dose levels both early and late in anaesthesia (Figure 1). At the deep level of anaesthesia, with ventilation controlled, the pressures obtained in the volunteers anaesthetized with isoflurane alone were 46 torr early and 53 torr late in anaesthesia, whereas the corresponding values from our isoflurane-nitrous oxide study were 72.5 and 75 torr. These higher pressures resulted from a higher total peripheral resistance. Heart rate, stroke volume, and cardiac output were similar in both studies.

The use of nitrous oxide also lessened the amount of hypotension seen with isollurane when ventilation was spontaneous (Table V). The improvement in pressure with nitrous oxide was not as great as seen with controlled ventilation, 
TABLE IV

Blood Volume and. HaEmatocrit Values

\begin{tabular}{lccc}
\hline \hline & Awake & $\begin{array}{c}\text { After 2 Hours } \\
\text { Anaesthesia }\end{array}$ & $\begin{array}{c}\text { After 6 Hours } \\
\text { Anaesthesia }\end{array}$ \\
\hline Blood Volume (liters) & $6.8 \pm 0.4$ & $6.6 \pm 0.4$ & $6.2 \pm 4$ \\
Haematocrit (per cent) & $43 \pm 1$ & $44 \pm 1$ & $42 \pm 1$ \\
\hline
\end{tabular}

$( \pm \mathrm{SE})$.

perhaps because vasodilatory properties of hypercapnia tended to counteract the alpha-sympathetic stimulation of nitrous oxide. ${ }^{9}$

Stevens, et al. ${ }^{6}$ found no evidence that isoflurane in oxygen depressed the heart directly during controlled ventilation. Even at deep levels of anaesthesia and rather profound hypotension, the cardiac output was maintained at control levels. Cromwell, et al. ${ }^{7}$ found that with spontaneous ventilation during isofluraneoxygen anaesthesia, cardiac output actually rose to 150 to 200 per cent of control without alteration of I-J amplitude or pre-ejection period. We found similar results for isoflurane-nitrous oxide anaesthesia.

Isoflurane-nitrous oxide anaesthesia is marked by the same high muscle blood flows seen with isoflurane alone. ${ }^{6, \pi}$ This high muscle blood flow is in contrast to all other inhalational anaesthetics studied, including halothane, cyclopropane, ether, and fluroxene where no significant increase in muscle blood flow was seen in the first hour of anaesthesia. ${ }^{15}$ Does this increased flow to muscles compromise other organ systems by diverting blood flow away from them? Our data suggests not. We found no systemic acidosis and no toxicity was uncovered by liver or kidney studies during and after anaesthesia in our healthy volunteers. ${ }^{10}$

\section{Respiratory Effects}

Addition of nitrous oxide failed to ameliorate the respiratory depression produced by isoflurane alone. Isoflurane-oxygen and isoflurane-nitrous oxide produced the same degree of hypercapnia at equivalent levels of anaesthesia (Figure 2). Arterial carbon dioxide tensions increased during isoflurane-nitrous oxide anaesthesia, despite a slight increase in minute ventilation engendered by a two and a half fold increase in respiratory rate at the light level of anaesthesia and a still faster rate at greater anaesthetic concentrations. In contrast, minute ventilation was less than control with isoflurane-oxygen anaesthesia and the respiratory rate was only twice normal over the range of doses studied. Hypercapnia in both groups resulted from shallow respirations and an increase in dead-space ventilation. The faster respiratory rate seen with isoflurane-nitrous oxide might have lessened the effective alveolar gas exchange with each breath by shortening the time for passive diffusion into and out of alveoli, ${ }^{17}$ perhaps explaining why the higher minute ventilation seen with isoflurane-nitrous oxide did not lessen the carbon dioxide retention.

Respiratory depression with isoflurane equals or exceeds that seen with all other inhalational anaesthetics. ${ }^{8}$ Since the concurrent use of 70 per cent nitrous oxide does not reduce this depression, the combination of isoflurane and nitrous oxide may produce hypoxia during spontaneous respiration. However, in our healthy volunteers, the average $\mathrm{PaO}_{2}$ at the deepest level of anaesthesia was 92 
TABLE $V$

Comparison of Results of Cardiovascular and Respiratory Measurements in Volunteers Receiving Light or Moderately DeEp Isoflurane-Nitrous Oxide or Isoflurane-Oxygen, Spontaneous or Controlled Ventilation

\begin{tabular}{|c|c|c|c|c|}
\hline \multirow[t]{2}{*}{ Number of Volunteers } & Spontaneous & $\begin{array}{l}\mathrm{N}_{2} \mathrm{O} \\
\mathrm{O}_{2}\end{array}$ & $\begin{array}{l}8 \\
9\end{array}$ & $\begin{array}{l}8 \\
9\end{array}$ \\
\hline & Controlled & $\begin{array}{l}\mathrm{N}_{2} \mathrm{O} \\
\mathrm{O}_{2}\end{array}$ & $\begin{array}{l}8 \\
7\end{array}$ & $\begin{array}{l}8 \\
7\end{array}$ \\
\hline \multirow[t]{2}{*}{$\begin{array}{l}\text { Time from Induction } \\
\text { (minutes) }\end{array}$} & Spontaneous & $\begin{array}{l}\mathrm{N}_{2} \mathrm{O} \\
\mathrm{O}_{2}\end{array}$ & $\begin{array}{l}189 \pm 14 \\
302 \pm 12\end{array}$ & $\begin{array}{l}213 \pm 5 \\
320 \pm 11\end{array}$ \\
\hline & Controlled & $\begin{array}{l}\mathrm{N}_{2} \mathrm{O} \\
\mathrm{O}_{2}\end{array}$ & $\begin{array}{l}334 \pm 4 \\
284 \pm 8\end{array}$ & $\begin{array}{l}367 \pm 4 \\
303 \pm 7\end{array}$ \\
\hline \multirow{2}{*}{$\begin{array}{l}\text { Concentration of } \\
\text { Anaesthetic } \\
\text { (end-tidal Forane) }\end{array}$} & Spontaneous & $\begin{array}{l}\mathrm{N}_{8} \mathrm{O} \dagger \\
\mathrm{O}_{2}\end{array}$ & $\begin{array}{l}1.38 \pm 0.01 \\
1.56 \pm 0.02\end{array}$ & $\begin{array}{l}2.02 \pm 0.02 \\
1.90 \pm 0.02\end{array}$ \\
\hline & Controlled & $\begin{array}{l}\mathrm{N}_{2} \mathrm{O} \dagger \\
\mathrm{O}_{2}\end{array}$ & $\begin{array}{l}1.39 \pm 0.01 \\
1.21 \pm 0.01^{*}\end{array}$ & $\begin{array}{l}2.04 \pm 0.02 \\
1.84 \pm 0.02\end{array}$ \\
\hline \multirow[t]{2}{*}{ MAC Equivalent } & Spontaneous & $\begin{array}{l}\mathrm{N}_{2} \mathrm{O} \\
\mathrm{O}_{2}\end{array}$ & $\begin{array}{l}1.09 \pm 0.01 \\
1.23 \pm 0.02\end{array}$ & $\begin{array}{l}1.59 \pm 0.02 \\
1.50 \pm 0.02\end{array}$ \\
\hline & Controlled & $\begin{array}{l}\mathrm{N}_{2} \mathrm{O} \\
\mathrm{O}_{2}\end{array}$ & $\begin{array}{l}1.10 \pm 0.10 \\
0.95 \pm 0.01\end{array}$ & $\begin{array}{l}1.60 \pm 0.02 \\
1.45 \pm 0.02\end{array}$ \\
\hline \multirow[t]{2}{*}{$\begin{array}{l}\text { Arterial Carbon } \\
\text { Dioxide Tension (torr) }\end{array}$} & Spontaneous & $\begin{array}{l}\mathrm{N}_{2} \mathrm{O} \\
\mathrm{O}_{2}\end{array}$ & $\begin{array}{l}47.3 \pm 1.9 \\
55.3 \pm 1.0^{*}\end{array}$ & $\begin{array}{l}58.5 \pm 3.2 \\
58.5 \pm 1.4\end{array}$ \\
\hline & Controlled & $\begin{array}{l}\mathrm{N}_{2} \mathrm{O} \\
\mathrm{O}_{2}\end{array}$ & $\begin{array}{l}39.5 \pm 0.7^{*} \\
37.7 \pm 0.8\end{array}$ & $\begin{array}{l}39.0 \pm 0.9^{*} \\
38.4 \pm 1.7^{*}\end{array}$ \\
\hline $\begin{array}{l}\text { Minute Ventilation } \\
\text { (liters/min) }\end{array}$ & Spontaneous & $\begin{array}{l}\mathrm{N}_{2} \mathrm{O} \\
\mathrm{O}_{2}\end{array}$ & $\begin{array}{c}7.6 \pm 0.3 \\
(n=7) \\
6.3 \pm 0.5\end{array}$ & $\begin{array}{c}6.8 \pm 0.4 \\
(n=7) \\
5.8 \pm 0.5\end{array}$ \\
\hline Minute Ventilation & Spontaneous & $\begin{array}{l}\mathrm{N}_{2} \mathrm{O} \\
\mathrm{O}_{2}\end{array}$ & $\begin{array}{l}113 \pm 7 \\
84 \pm 7^{*}\end{array}$ & $\begin{array}{c}100 \pm 8 \\
(\mathrm{n}=7) \\
77 \pm 7^{*}\end{array}$ \\
\hline Respiratory Rate & Spontaneous & $\begin{array}{l}\mathrm{N}_{2} \mathrm{O} \\
\mathrm{O}_{2}\end{array}$ & $\begin{array}{l}250 \pm 50 \\
171 \pm 7\end{array}$ & $\begin{array}{l}330 \pm 70 \\
178 \pm 7^{*}\end{array}$ \\
\hline Tidal Volume & Spontaneous & $\begin{array}{l}\mathrm{N}_{2} \mathrm{O} \\
\mathrm{O}_{2}\end{array}$ & $\begin{array}{l}54 \pm 8 \\
(\mathrm{n}=7) \\
48 \pm 3\end{array}$ & $\begin{array}{l}40 \pm 6 \\
(\mathrm{n}=7) \\
43 \pm 2\end{array}$ \\
\hline $\begin{array}{l}\text { Alveolar Ventilation } \\
\text { (liters/min) }\end{array}$ & Spontaneous & $\begin{array}{l}\mathrm{N}_{2} \mathrm{O} \\
\mathrm{O}_{2}\end{array}$ & $\begin{array}{c}2.4 \pm 0.1 \\
(n=7) \\
2.2 \pm 0.2\end{array}$ & $\begin{array}{c}1.0 \pm 0.2 \\
(\mathrm{n}=7) \\
2.0 \pm 0.2\end{array}$ \\
\hline$V_{D} / V_{T}$ (liters/liter) & Spontaneous & $\begin{array}{l}\mathrm{N}_{2} \mathrm{O} \\
\mathrm{O}_{2}\end{array}$ & $\begin{array}{c}0.68 \pm 0.20 \\
(\mathrm{n}=7) \\
0.65 \pm 0.02\end{array}$ & $\begin{array}{c}0.70 \pm 0.02 \\
(n=7) \\
0.65 \pm 0.02\end{array}$ \\
\hline \multirow[t]{2}{*}{$\begin{array}{l}\text { Mean Arterial } \\
\text { Blood Pressure }\end{array}$} & Spontaneous & $\begin{array}{l}\mathrm{N}_{2} \mathrm{O} \\
\mathrm{O}_{2}\end{array}$ & $\begin{array}{l}81 \pm 5 \\
72 \pm 4\end{array}$ & $\begin{array}{l}79 \pm 4 \\
68 \pm 3^{*}\end{array}$ \\
\hline & Controlled & $\begin{array}{l}\mathrm{N}_{2} \mathrm{O} \\
\mathrm{O}_{2}\end{array}$ & $\begin{array}{l}88 \pm 5 \\
71 \pm 3^{*}\end{array}$ & $\begin{array}{l}76 \pm 3 \\
60 \pm 3^{*}\end{array}$ \\
\hline \multirow[t]{2}{*}{ Cardiac Output } & Spontaneous & $\begin{array}{l}\mathrm{N}_{2} \mathrm{O} \\
\mathrm{O}_{2}\end{array}$ & $\begin{array}{l}116 \pm 5 \\
122 \pm 7\end{array}$ & $\begin{array}{l}142 \pm 10 \\
138 \pm 7\end{array}$ \\
\hline & Controlled & $\begin{array}{l}\mathrm{N}_{2} \mathrm{O} \\
\mathrm{O}_{2}\end{array}$ & $\begin{array}{l}107 \pm 6 \\
101 \pm 7\end{array}$ & $\begin{array}{l}107 \pm 2^{*} \\
102 \pm 9^{*}\end{array}$ \\
\hline \multirow[t]{2}{*}{$\begin{array}{l}\text { Total Peripheral } \\
\text { Resistance }\end{array}$} & Spontaneous & $\begin{array}{l}\mathrm{N}_{2} \mathrm{O} \\
\mathrm{O}_{2}\end{array}$ & $\begin{array}{l}75 \pm 7 \\
60 \pm 3\end{array}$ & $\begin{array}{l}57 \pm 5 \\
50 \pm 2\end{array}$ \\
\hline & Controlled & $\begin{array}{l}\mathrm{N}_{2} \mathrm{O} \\
\mathrm{O}_{2}\end{array}$ & $\begin{array}{l}84 \pm 5 \\
73 \pm 6\end{array}$ & $\begin{array}{l}71 \pm 4^{*} \\
62 \pm 7\end{array}$ \\
\hline
\end{tabular}


TABLE V-continued

\begin{tabular}{|c|c|c|c|c|}
\hline \multirow[t]{2}{*}{$\begin{array}{l}\text { Base Deficit } \\
\text { (mEq change from control) }\end{array}$} & Spontaneous & $\begin{array}{l}\mathrm{N}_{2} \mathrm{O} \\
\mathrm{O}_{2}\end{array}$ & $\begin{array}{r}1.6 \pm 0.7 \\
-0.6 \pm 0.8\end{array}$ & $\begin{array}{l}1.9 \pm 0.9 \\
0.0 \pm 0.7\end{array}$ \\
\hline & Controlled & $\begin{array}{l}\mathrm{N}_{2} \mathrm{O} \\
\mathrm{O}_{2}\end{array}$ & $\begin{array}{r}-0.1 \pm 0.8 \\
0.3 \pm 0.4\end{array}$ & $\begin{array}{l}0.2 \pm 0.6 \\
0.2 \pm 0.5\end{array}$ \\
\hline \multirow{3}{*}{$\begin{array}{l}\text { Minute Oxygen } \\
\text { Consumption }\end{array}$} & Spontaneous & $\mathrm{N}_{2} \mathrm{O}$ & $75 \pm 7$ & $76 \pm 7$ \\
\hline & & $\mathrm{O}_{2}$ & $83 \pm 10$ & $82 \pm 10$ \\
\hline & Controlled & $\begin{array}{l}\mathrm{N}_{2} \mathrm{O} \\
\mathrm{O}_{2}\end{array}$ & $\begin{array}{l}76 \pm 7 \\
87 \pm 12\end{array}$ & $\begin{array}{l}67 \pm 7 \\
72 \pm 11\end{array}$ \\
\hline \multirow[t]{2}{*}{$\begin{array}{l}\text { Cardiac Output/Oxygen } \\
\text { Consumption Ratio }\end{array}$} & Spontaneous & $\begin{array}{l}\mathrm{N}_{2} \mathrm{O} \\
\mathrm{O}_{2}\end{array}$ & $\begin{array}{l}158 \pm 13 \\
158 \pm 17\end{array}$ & $\begin{array}{l}201 \pm 26 \\
152 \pm 12\end{array}$ \\
\hline & Controlled & $\begin{array}{l}\mathrm{N}_{2} \mathrm{O} \\
\mathrm{O}_{2}\end{array}$ & $\begin{array}{l}146 \pm 10 \\
123 \pm 12\end{array}$ & $\begin{array}{l}169 \pm 17 \\
127 \pm 12^{*}\end{array}$ \\
\hline \multirow[t]{2}{*}{$\begin{array}{l}\text { Forearm (muscle) } \\
\text { Blood Flow }\end{array}$} & Spontaneous & $\begin{array}{l}\mathrm{N}_{2} \mathrm{O} \\
\mathrm{O}_{2}\end{array}$ & $\begin{array}{l}292 \pm 37 \\
417 \pm 74\end{array}$ & $\begin{array}{l}334 \pm 62 \\
426 \pm 45\end{array}$ \\
\hline & Controlled & $\begin{array}{l}\mathrm{N}_{2} \mathrm{O} \\
\mathrm{O}_{2}\end{array}$ & $\begin{array}{l}361 \pm 54 \\
302 \pm 46\end{array}$ & $\begin{array}{l}347 \pm 52 \\
334 \pm 69\end{array}$ \\
\hline \multirow[t]{2}{*}{$\begin{array}{l}\text { Finger (skin) } \\
\text { Blood Flow }\end{array}$} & Spontaneous & $\begin{array}{l}\mathrm{N}_{2} \mathrm{O} \\
\mathrm{O}_{2}\end{array}$ & $\begin{array}{l}182 \pm 51 \\
423 \pm 158\end{array}$ & $\begin{array}{l}165 \pm 40 \\
397 \pm 88^{*}\end{array}$ \\
\hline & Controlled & $\begin{array}{l}\mathrm{N}_{2} \mathrm{O} \\
\mathrm{O}_{2}\end{array}$ & $\begin{array}{l}152 \pm 31 \\
304 \pm 70\end{array}$ & $\begin{array}{l}141 \pm 32 \\
271 \pm 85\end{array}$ \\
\hline \multirow[t]{2}{*}{$\begin{array}{l}\text { Oesophageal Temperature } \\
\left({ }^{\circ} \mathrm{C}\right)\end{array}$} & Spontaneous & $\begin{array}{l}\mathrm{N}_{2} \mathrm{O} \\
\mathrm{O}_{2}\end{array}$ & $\begin{array}{l}36.9 \pm 0.1 \\
36.9 \pm 0.1\end{array}$ & $\begin{array}{l}36.9 \pm 0.1 \\
36.9 \pm 0.1\end{array}$ \\
\hline & Controlled & $\begin{array}{l}\mathrm{N}_{2} \mathrm{O} \\
\mathrm{O}_{2}\end{array}$ & $\begin{array}{l}36.9 \pm 0.1 \\
36.7 \pm 0.1\end{array}$ & $\begin{array}{l}36.9 \pm 0.1 \\
36.7 \pm 0.1\end{array}$ \\
\hline \multirow[t]{2}{*}{ Heart Rate } & Spontaneous & $\begin{array}{l}\mathrm{N}_{2} \mathrm{O} \\
\mathrm{O}_{2}\end{array}$ & $\begin{array}{l}124 \pm 6 \\
148 \pm 10\end{array}$ & $\begin{array}{l}132 \pm 7 \\
150 \pm 9\end{array}$ \\
\hline & Controlled & $\begin{array}{l}\mathrm{N}_{2} \mathrm{O} \\
\mathrm{O}_{2}\end{array}$ & $\begin{array}{l}138 \pm 8 \\
119 \pm 9\end{array}$ & $\begin{array}{l}132 \pm 6 \\
125 \pm 8\end{array}$ \\
\hline \multirow[t]{2}{*}{ Stroke Volume } & Spontaneous & $\begin{array}{l}\mathrm{N}_{2} \mathrm{O} \\
\mathrm{O}_{2}\end{array}$ & $\begin{array}{l}96 \pm 7 \\
83 \pm 7\end{array}$ & $\begin{array}{r}109 \pm 8 \\
92 \pm 6\end{array}$ \\
\hline & Controlled & $\begin{array}{l}\mathrm{N}_{2} \mathrm{O} \\
\mathrm{O}_{2}\end{array}$ & $\begin{array}{l}79 \pm 5 \\
87 \pm 5\end{array}$ & $\begin{array}{l}80 \pm 4^{*} \\
82 \pm 5^{*}\end{array}$ \\
\hline \multirow[t]{2}{*}{$\begin{array}{l}\text { Left Ventricular } \\
\text { Minute Work }\end{array}$} & Spontaneous & $\begin{array}{l}\mathrm{N}_{2} \mathrm{O} \\
\mathrm{O}_{2}\end{array}$ & $\begin{array}{l}94 \pm 5 \\
87 \pm 8\end{array}$ & $\begin{array}{r}125 \pm 18 \\
91 \pm 10\end{array}$ \\
\hline & Controlled & $\begin{array}{l}\mathrm{N}_{2} \mathrm{O} \\
\mathrm{O}_{2}\end{array}$ & $\begin{array}{l}94 \pm 8 \\
73 \pm 5^{*}\end{array}$ & $\begin{array}{l}81 \pm 6^{*} \\
65 \pm 5^{*}\end{array}$ \\
\hline \multirow[t]{3}{*}{ Pre-ejection Period } & Spontaneous & $\begin{array}{l}\mathrm{N}_{2} \mathrm{O} \\
\mathrm{O}_{2}\end{array}$ & $\begin{array}{l}96 \pm 17 \\
79 \pm 11\end{array}$ & $\begin{array}{l}99 \pm 25 \\
77 \pm 13\end{array}$ \\
\hline & Controlled & $\mathrm{N}_{2} \mathrm{O}$ & $\begin{array}{c}118 \pm 18 \\
(n=7)\end{array}$ & $\begin{array}{c}113 \pm 14 \\
(n=7)\end{array}$ \\
\hline & & $\mathrm{O}_{2}$ & $\begin{array}{l}92 \pm 25 \\
(n=5)\end{array}$ & $\begin{array}{c}86 \pm 13 \\
(\mathrm{n}=6)\end{array}$ \\
\hline \multirow[t]{2}{*}{ I-J Wave Amplitude } & Spontaneous & $\begin{array}{l}\mathrm{N}_{2} \mathrm{O} \\
\mathrm{O}_{2}\end{array}$ & $\begin{array}{r}114 \pm 11 \\
83 \pm 5^{*}\end{array}$ & $\begin{array}{c}120 \pm 16 \\
92 \pm 8\end{array}$ \\
\hline & Controlled & $\begin{array}{l}\mathrm{N}_{2} \mathrm{O} \\
\mathrm{O}_{2}\end{array}$ & $\begin{array}{l}99 \pm 13 \\
91 \pm 17 \\
(n=5)\end{array}$ & $\begin{array}{l}95 \pm 12 \\
95 \pm 21 \\
(\mathrm{n}=5)\end{array}$ \\
\hline \multirow{2}{*}{$\begin{array}{l}\text { Mean Right Atrial } \\
\text { Pressure (torr change } \\
\text { from control) }\end{array}$} & Spontaneous & $\begin{array}{l}\mathrm{N}_{2} \mathrm{O} \\
\mathrm{O}_{2}\end{array}$ & $\begin{array}{r}-2.2 \pm 1.1 \\
0.0 \pm 0.6\end{array}$ & $\begin{array}{r}-1.3 \pm 1.0 \\
0.3 \pm 0.6\end{array}$ \\
\hline & Controlled & $\begin{array}{l}\mathrm{N}_{2} \mathrm{O} \\
\mathrm{O}_{2}\end{array}$ & $\begin{array}{r}-1.9 \pm 0.8 \\
0.0 \pm 0.6\end{array}$ & $\begin{array}{r}-0.9 \pm 0.9 \\
0.0 \pm 0.3\end{array}$ \\
\hline
\end{tabular}

Values are given as per cent of control unless otherwise indicated. ${ }^{*}=$ significant difference between isoflurane-nitrous oxide spontaneous ventilation and the other three categories. $\dagger=$ anaesthetic concentration of isoflurane plus nitrous oxide was obtained by adding 0.75 to the endtidal isoflurane per cent concentration. 

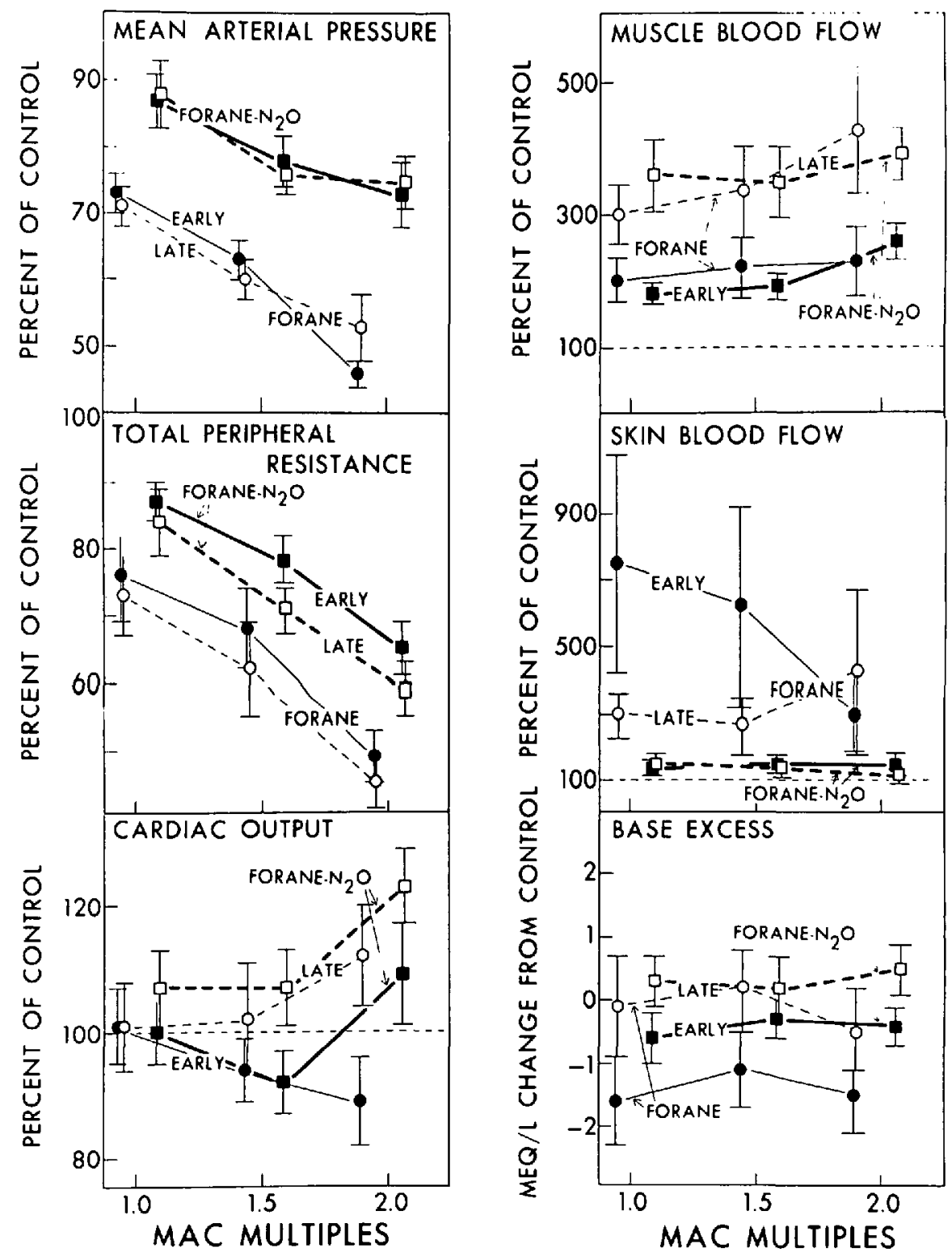

Figure 1. A comparison of isoflurane-nitrous oxide and isoflurane-oxygen anaesthesia during controlled ventilation in their effects on cardiovascular variables. Mean values \pm 1 standard error are shown.

torr. In addition, the finding that surgical stimulation tends to increase respiration would oppose any tendency to a lower arterial oxygen level. ${ }^{18}$

\section{RÉSUMÉ}

Nous avons étudié les effets cardio-respiratoires de l'anesthésie à l'isoflurane (Forane) associé à un mélange de Protoxyde-Oxygène à 70 pour cent. Cette étude fut réalisée chez huit sujets volontaires, en bonne santé, en respiration contrôlée $\left(\mathrm{PaCO}_{2} 35-45\right.$ torr $)$ et en respiration spontanée. Nos résultats furent comparés à ceux rapportés dans d'autres études effectuées chez des volontaires avec usage d'isoflurane-oxygène. 
DOLAN, et al.: ISOFLURANE-NITROUS OXIDE ANAESTHESIA

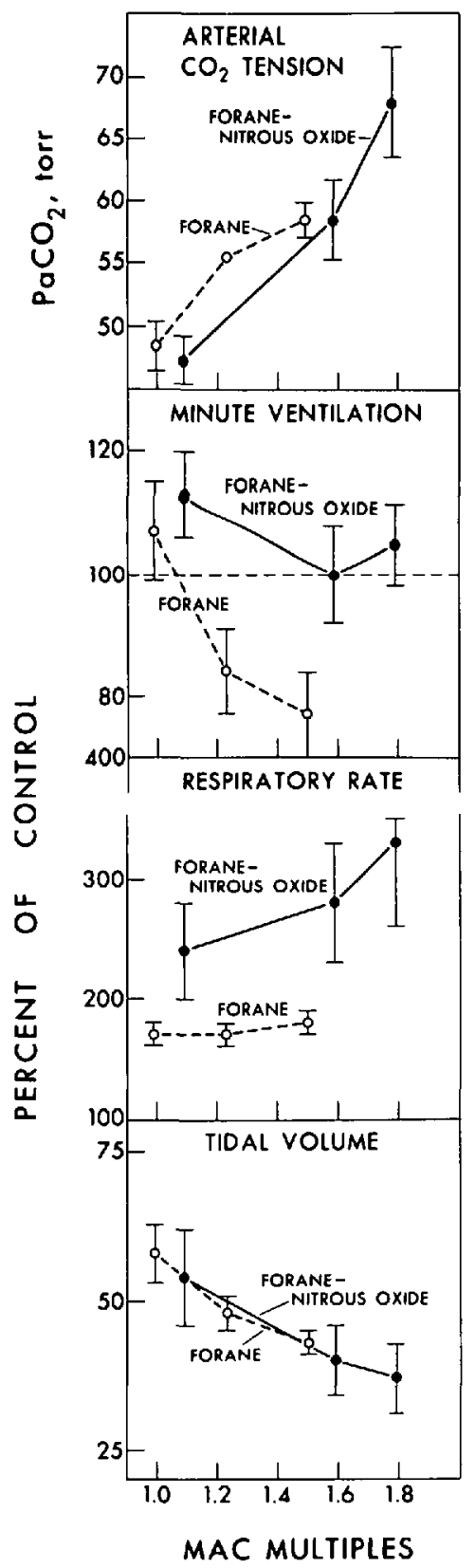

Figure 2. A comparison of isoflurane-nitrous oxide and isoflurane-oxygen in their effects on respiratory variables. Mean values \pm 1 standard error are shown.

Sous respiration contrôlée, la pression artérielle moyenne était plus élevée de 10 à 25 pour cent chez les malades recevant du Protoxyde. Sous anesthésie profonde (2 MAC environ) on retrouvait une pression moyenne de 72.5 torr dans le groupe avec usage de Protoxyde, alors qu'un chiffre moyen de 46 torr était obtenu avec usage d'Isoflurane-Oxygène.

Tout comme chez les sujets endormis à l'Isoflurane-oxygène, nous avons observé chez nos sujets un bon maintien de la contractilité myocardique et du débit car- 
diaque, ceci à tous les niveaux d'anesthésie utilisés, et l'on n'a pas observé d'acidose métabolique.

Le débit sanguin musculaire était de deux à quatre fois plus élevé qu'avant l'anesthésie.

Des différences cardiovasculaires dans le même sens ont été observées chez les malades en respiration spontanée.

Cependant, la présence de Protoxyde d'azote n'a pas modifié la dépression respiratoire observée avec l'usage d'isoflurane-oxygène, à une profondeur de 1.5 $\mathrm{MAC}$, en respiration spontanée, les $\mathrm{PaCO}_{2}$ moyens observés étaient de $58 \pm 3$ torr. avec Protoxyde et de $58 \pm 1.4$ sans Protoxyde.

\section{ACKNOWLEDGMENTS}

The authors greatly appreciate the able assistance of Mr. Richard Shargel, Mrs. Anne White, Miss Eunice Lim, and Miss Judy MacLean in the completion of this study.

\section{REFERENCES}

1. Vitcha, J.F. A history of Forane (editorial). Anesthesiology 35: 4-7 (1971).

2. Cromwell, T.H., Eger, E.I. II, Stevens, W.C., et al. Forane uptake, excretion, and blood solubility in man. Anesthesiology 35: 401-408 (1971).

3. Halsey, M.J., SAwyer, D.C., Ecer, E.I. II, et al. Hepatic metabolism of halothane, methoxyflurane, cyclopropane, Ethrane, and Forane in miniature swine. Anesthesiology 35: 43-47 (1971).

4. Joas, T.A. \& Stevens, W.C. Comparison of the arrhythmic doses of epinephrine during Forane, halothane, and fluroxene anesthesia in dogs. Anesthesiology 35: 48-53 (1971).

5. Miller, R.D., Eger, E.I. II, War, W.L., et al. Comparative neuromuscular effects of Forane and halothane alone and in combination with d-tubocurarine in man. Anesthesiology 35: 38-42 (1971).

6. Stevens, W.C., Cromwell, T.H., Halsey, M.J., et al. The cardiovascular effects of a new inhalation anesthetic, Forane, in human volunteers at constant end-tidal carbon dioxide tension. Anesthesiology 35: 8-16 (1971).

7. Chomwell, T.H., Stevens, W.C., EGer, E.I. II, et al. The cardiovascular effects of compound 469 (Forane) during spontaneous ventilation and $\mathrm{CO}_{2}$ challenge in man. Anesthesiology 35: 17-25 (1971).

8. Fourcade, H.F., Stevens, W.C., Lanson, C.P. Jr., et al. Ventilatory effects of Forane a new inhalational anesthetic. Anesthesiology 35: 26-31 (1971).

9. Honnein, T.F., Martin, W.E., \& Bonica, J.J, Nitrous oxide effects on the circulatory and ventilatory responses to halothane. Anesthesiology 31: 250-260 (1969).

10. Smith, N.T., Ecer, E.I. II, Stoeltinc, R.K., et al. The cardiovascular and sympathomimetic responses to the addition of nitrous oxide to halothane in man. Anesthesiology 32: 410-421 (1970).

11. Bahlman, S.H., Ecer, E.I. II, SMith, N.T., et al. The cardiovascular effects of nitrous oxide-halothane anesthesia in man. Anesthesiology 35: 274-285 (1971).

12. Eger, E.I. II SMith, N.T., Stoeltivg, R.K, et al. Cardiovascular effects of halothane in man. Anesthesiology 32: 392-409 (1970).

13. Severinghaus, J.W. Continuously recording ventimeter. Anesthesiology 23: 482-483 (1962).

14. Stevens, W.C., Eger, E.I. II, Dolan, W.M., et al. The minimum alveolar concentration of a new inhalational anesthetic, Forane, in man. Abstract, American Society of Anesthesiologists, Atlanta, October, 1971, p. 181.

15. EgER, E.I. II. A comparison of the cardiovascular effects of halothane, fluroxene, cyclopropane, and ether in man. Anesthesiology 34: 25-41 (1971).

16. Stevens, W.C., Ecer, E.I. II, JoAs, T.A., et al. Comparative toxicity of isoflurane, halothane, fluroxene, and diethyl ether in human volunteers. Canad. Anaesth. Soc. J. 20: $357-376$ (1973).

17. Georg, J., Lassen, N.A., Mrllengrand, K, et al. Diffusion in the gas phase of the lungs in normal and emphysematous subjects. Clinical Science 29: 525-5.32 (1965).

18. Eger, E.I. II, Dolan, W.M., Stevens, W.C., et al. Surgical stimulation antagonizes the respiratory depression produced by Forane. Anesthesiology 36:544-549 (1972). 